\title{
HTF9C gene of 22q11.21 region associates with schizophrenia having deficit-sustained attention
}

\author{
Yu-Li Liu ${ }^{a}$, Cathy Shen-Jang Fann ${ }^{c}$, Chih-Min Liu ${ }^{b}$, Chien Ching Chang ${ }^{c}$, Wei- \\ Chih Yang ${ }^{c}$, Jer-Yuarn Wu ${ }^{d}$, Shuen-lu Hung ${ }^{d}$, Hung-Yu Chan ${ }^{\mathrm{e}}$, Jiahn-Jyh Chen ${ }^{\mathrm{e}}$, \\ Ming H. Hsieh ${ }^{\mathrm{b}}$, Tzung-Jeng Hwang ${ }^{\mathrm{b}}$, Stephen V. Faraone ${ }^{\mathrm{h}}$, Ming T. Tsuang ${ }^{\mathrm{i}}$, \\ Wei J. Chen ${ }^{f}$ and Hai-Gwo Hwu ${ }^{\text {b,f,g }}$
}

\begin{abstract}
Objective A region at chromosome 22q11.21 has been reported to potentially harbor a candidate gene for schizophrenia, ZDHHC8 (zinc finger, DHHC domain containing 8; also annotated as KIAA1292) in a number of studies. This finding has been replicated in Han Chinese, but not in other ethnicity-specific studies. For further support from within the Han Chinese ethnic group, we selected two single nucleotide polymorphisms (SNP) located at the distal $5^{\prime}$-end (rs1633445; intron 10 of Hpall tiny fragments locus 9C, HTF9C) and the intron 4 (rs175174) of ZDHHC8 gene to test if these were associated with schizophrenia in a study sample of Taiwan.

Methods A total of 218 schizophrenia families with at least two affected siblings participated in this study. These two SNPs were genotyped using matrix-assisted laser desorption/localization ionization time of flight (MALDI-TOF) mass spectrometry.
\end{abstract}

Results Significant associations with schizophrenia were not shown from these two SNPs. After stratifying schizophrenia according to the deficit and the nondeficit of sustained attention assessed by the Continuous Performance Test, the rs 1633445 showed significant association with schizophrenia in the presence of a deficit in sustained attention $(P<0.04)$.

\section{Introduction}

Significant association between a region of microdeletions at chromosome 22q11.21 and schizophrenia has been reported in a number of studies (Karayiorgou and Gogos, 2004; Horowitz et al., 2005). Notable, the ZDHHC8 gene (zinc finger, DHHC domain containing 8) located in this region has been studied as a candidate gene for schizophrenia, based upon the finding of a single nucleotide polymorphism (SNP) rs175174 located at intron 4 (Mukai et al., 2004). Functionally, this SNP has transmission preference in women, and may alter genetic splicing as well as modulate levels of ZDHHC8 expression at different allele types. Additionally, in ZDHHC8 knockout mice, Mukai et al. observed alterations in the sensorimotor gating of prepulse inhibition, and exploratory activity of locomotor response to novelty. To further
Conclusion SNP rs1633445 of the HTF9C gene may be associated with a deficit in sustained attention within schizophrenia, in a Taiwanese cohort. The deficit of sustained attention may be an endophenotype of schizophrenia, and warrants further study Psychiatr Genet 17:333-338 ๔ 2007 Wolters Kluwer Health | Lippincott Williams \& Wilkins.

Psychiatric Genetics 2007, 17:333-338

Keywords: 22q11.21, HTF9C, schizophrenia, sustained attention, ZDHHC8

aDivision of Mental Health and Substance Abuse Research, National Health Research Institute, Taiwan, ${ }^{\mathrm{b}}$ Department of Psychiatry, National Taiwan University Hospital and National Taiwan University College of Medicine, 'Institute of Biomedical Sciences, dNational Genotyping Center, Institute of Biomedical Sciences, Academia Sinica, Taipei, ' $T a o y u a n$ Psychiatric Center, Taoyuan, Institute of Epidemiology, College of Public Health, ${ }^{\mathrm{f}}$ Department of Psychology, College of Science, National Taiwan University, Taipei, Taiwan, ${ }^{h}$ Medical Genetics Research Center and Department of Psychiatry and Behavioral Sciences, SUNY Upstate Medical University, Syracuse, New York and 'Harvard Institute of Psychiatric Epidemiology and Genetics and Departments of Epidemiology and Psychiatry, Harvard University, Boston, Massachusetts and Institute of Behavioral Genomics, University of California, San Diego, California, USA

Correspondence to Chung-Shan, MD, Department of Psychiatry, National Taiwan University Hospital, No. 7, 10002 South Road, Taipei, Taiwan, 100 Tel: +886223123456 (ext) 6657; fax: +88622375 3663; e-mail: haigohwu@ntu.edu.tw

Received 14 May 2006 Revised 12 December 2006 Accepted 21 December 2006

explore and predict the function of this novel gene, a homology search of different species found that there are two yeast ZDHHC proteins demonstrating as the enzyme of palmitoyltransferase (Lobo et al., 2002; Roth et al., 2002). Palmitoyltransferase is involved in neuronal development and function (El-Husseini Ael and Bredt, 2002), suggesting that the ZDHHC8 gene may be involved in the pathological process of schizophrenia.

ZDHHC8 has been investigated as a schizophrenia candidate gene in both case-control studies and proband-parent triads, with negative results reported in European (Glaser et al., 2005), Japanese (Saito et al., 2005), and German Caucasian (Faul et al., 2005) ethnic groups, and supportive evidence found solely in the Han Chinese (Chen et al., 2004b) ethnic group. As 
ZDHHC8 has not been studied in the population of Taiwan, other supporting evidence is essential from other ethnic groups to substantiate that the ZDHHC8 gene and its located region confers true schizophrenia susceptibility.

Heterogeneity of schizophrenia will also contribute to the inconsistent association findings of schizophrenia and ZDHHC8. The deficit in sustained attention measured by the continuous performance test (CPT) (Rosvold et al., 1956) presents in schizophrenic patients, as well as in patients with schizotypal personality disorder and in nonpsychotic relatives of schizophrenic patients (Cornblatt and Keilp, 1994; Chen and Faraone, 2000). Using 2.5 standard deviations or more below the population mean as threshold, the recurrence risk ratio for CPT performance among parents or siblings was higher than that of schizophrenia alone (Chen et al., 2004a; Liu et al., 2006). This suggests that a deficit in CPT performance at this level could be a valid endophenotype for schizophrenia. We hypothesized that a significant association exists between the specific candidate gene and a proportion of schizophrenia with the defined endophenotypic indicator.

This study examined two independent hypotheses: (i) An association of schizophrenia exists, as a whole, and the ZDHHC8 genetic region covering the distal 5 '-end of another gene (HTF9C) by genotyping two SNP markers on 218 Taiwanese multiplex families (i.e. with at least two siblings affected with schizophrenia). (ii) An association of ZDHHC8 genetic polymorphism exists with the subgroup of schizophrenia defined by the deficit of sustained attention, which has been shown to be an endophenotype for schizophrenia in our previous study (Chen et al., 2004a).

\section{Methods}

This research project was approved by the Institutional Review Board of the National Taiwan University Hospital. Signed informed consent was obtained from all study subjects, who then underwent diagnostic assessment and collection of DNA samples. The participants were recruited from two sample-collecting programs, the 1993-2001 Multidimensional Psychopathology Study of Schizophrenia (MPSS) (Hwu et al., 2002) and the 19982002 Taiwan Schizophrenia Linkage Study (TSLS) (Hwu et al., 2005). The 86 MPSS families were interviewed by research psychiatrists using the Psychiatrist Diagnostic Assessment instrument (Hwu, 1999). The 132 TSLS families were interviewed by well-trained assistants using the Mandarin Chinese version of the Diagnostic Interview for Genetic Studies (Chen, 1999). The final diagnostic assessments for both MPSS and TSLS were formulated by integrating the Psychiatrist Diagnostic Assessment or Diagnostic Interview for Genetic Studies
Table 1 Distribution of families by number of siblings and parents genotyped

\begin{tabular}{lcrrr}
\hline & \multicolumn{3}{c}{ Parents genotyped per family } & \\
\cline { 2 - 4 } Sibs genotyped per family & \multicolumn{1}{c}{0} & \multicolumn{1}{c}{1} & \multicolumn{1}{c}{2} & Total families \\
\hline 1 & 0 & 0 & 2 & 2 \\
2 & 9 & 15 & 74 & 98 \\
3 & 8 & 78 & 17 & 103 \\
4 & 2 & 9 & 2 & 13 \\
5 & 0 & 1 & 1 & 2 \\
Total families & 19 & 103 & 96 & 218 \\
\hline
\end{tabular}

data with the clinical information obtained from medical chart records using the checking list of Specialist Diagnostic Assessment Sheet, on the basis of the relevant criteria of the Diagnostic and Statistical Manual of Mental Disorder, 4th edition (DSM-IV) (American Psychiatric Association, 1994). This study sample included 218 schizophrenic nuclear families with at least two affected siblings, which include 434 probands, 569 sib participants, and 295 parent participants (Table 1). A total of 864 participants participated in this genotyping study.

A CPT machine from Sunrise System, v. 2.20 (Pembroke, Massachusetts, USA), was used to assess sustained attention. The procedure has been described in detail elsewhere (Chen et al., 1998). Briefly, numbers from 0 to 9 were randomly presented for $50 \mathrm{~ms}$ each, at a rate of one per second. Each participant undertook two CPT sessions: the undegraded 1-9 task and the $25 \%$ degraded 1-9 task. Participants were asked to respond whenever the number ' 9 ' preceded by the number ' 1 ' appeared on the screen. A total of 331 trials, 34 (10\%) of which were target stimuli, were presented over $5 \mathrm{~min}$ for each session. During the $25 \%$ degraded session, a pattern of snow was used to toggle background and foreground so that the image was visually distorted. Each test session began with 2 min of practice (repeated if participants required). One signal-detection index of performance on the test, sensitivity $\left(\mathrm{D}^{\prime}\right)$, was derived from the hit rate (probability of response to target trials) and false-alarm rate (probability of response to nontarget trials) (Nuechterlein, 1991). Sensitivity is an individual's ability to discriminate target stimuli from nontarget stimuli. In a 1-week test-retest reliability study (Chen et al., 1998) of the CPT versions used in this study, the intraclass correlation coefficients or reliability of $\mathrm{D}^{\prime}$ were 0.83 and 0.82 for the undegraded and the $25 \%$ degraded 1-9 task, respectively.

The participants' CPT raw scores were standardized against the community norm, which contained 345 adults randomly sampled from a community (Chen et al., 1998), with adjustment for demographic features to provide adjusted $z$ scores. As age, sex, and educational level have been demonstrated to influence the CPT performance 
in the community sample, the predictive score of a participant was calculated by using the regression coefficients obtained from the regression of the scores on the three covariates among the 345 community participants. The difference between the raw score and the predictive score was then standardized by the root mean error of the regression and was defined as the adjusted $z$ score of the participant. The adjusted $z$ scores thus provide an estimate of the magnitude of deviation from the community norm. The study families were classified into attention deficit and nondeficit groups. If one of the affected siblings in the family has $z$ score of sensitivity indicator $\mathrm{D}^{\prime}$ lower than -2.5 , the family is classified as a CPT deficit family. Otherwise, the others are nondeficit families. Both the $z$ score of $\mathrm{D}^{\prime}$ of undegraded and degraded CPT were used for defining the CPT deficit family. The rationale for choosing the -2.5 threshold comes from our previous family study for CPT (Chen et al., 2004a; Liu et al., 2006), which showed that as the cut-off point in the adjusted $\approx$ score decreased from -2.5 to -3.0 , the risk ratio increased continually for both the undegraded (10.1-18.8 for parents, 10.0-16.7 for siblings) and degraded (12.4102.7 for parents, 8.6-72.0 for siblings) test. Therefore, using 2.5 standard deviations or more below the population mean as threshold, the recurrence risk ratio for CPT performance among parents or siblings was higher than that of schizophrenia alone.

The ZDHHC8 gene SNP (rs175174) and its distal 5'-end SNP (rs1633445) of HTF9C gene were genotyped by using Matrix-assisted laser desorption/ionization-time of flight mass spectrometry (MALDI-TOF MS). A DNA fragment (100-300 bp) encompassing the SNP site was amplified using the polymerase chain reaction (PCR) GeneAmp 9700 thermocycler (Applied Biosystems, Foster City, California, USA), according to manufacturer's instructions. After PCR amplification and neutralization of the deoxynucleotide triphosphate (dNTP), primer extension was performed by adding the probe Thermo Sequenase (Amersham Pharmacia, Piscataway, New Jersey, USA), and the appropriate dideoxynucleotide triphosphate (ddNTP)/dNTP mixture. The various extension products were differentiated by mass using MALDI-TOF spectrometry.
Genotyping data quality was checked on the indicators of Hardy-Weinberg equilibrium, the lineage of the study families, and the deviation from Mendelian inheritance by using the statistical programs of the ALLELE procedure in the SAS/GENETICS, (Cary, North Carolina, USA), the PEDCHECK (O'Connell and Weeks, 1998), and the UNKNOWN respectively. The haplotype blocks were constructed by the Haploview software for haplotype blocks constituted by 'strong LD' markers according to the criteria proposed by Gabriel et al. (2002). The phenotype of schizophrenia was divided into narrow model (DSM-IV defined schizophrenia only) and broad model (including schizophrenia, schizoaffective, and other nonaffective psychotic disorders defined by DSMIV) for the association study. Association tests of schizophrenia and the two SNP markers, both as single points and haplotypes, were carried out simultaneously using the TRANSMIT (Clayton, 1999) and the TRANSMIT and FBAT were free available software and were developed by the authors of Clayton (1999) and Horvath et al. (2004) listed in reference. The single point association analysis was also performed using the subgroups with attention deficit families.

\section{Results}

The SNP markers, rs175174 and rs1633445, were evaluated for their associations with schizophrenia (Table 2). Validation of these SNPs revealed that the minor allele frequency was above $15 \%$ and the missinggenotyping rate below 2\%; with distribution of these two SNP markers in Hardy-Weinberg equilibrium. A haplotype block with two SNP markers was generated using Haploview software (Gabriel et al., 2002), which creates a block when $95 \%$ of informative comparisons are strongly linked and the span is no more than $30 \mathrm{~kb}$. The linkage disequilibrium $\left(\mathrm{D}^{\prime}\right)$ for this haplotype block was only 0.24 for the two markers, and thus, the haplotype of these two SNPs was not appropriate in genetic analyses.

No significant association was found for any single SNP marker of the ZDHHC8 or HTF9C gene using both narrow and broad phenotype models of schizophrenia (Table 2). The model-specific transmission to nontransmission ratios for each SNP were 1.0124 (broad

Table 2 Frequencies of the single nucleotide polymorphisms of the ZDHHC8 gene and association with two phenotype models of schizophrenia

\begin{tabular}{|c|c|c|c|c|c|c|c|c|c|c|c|}
\hline \multirow[b]{2}{*}{ SNP_ID } & \multirow[b]{2}{*}{ Chromosome } & \multirow[b]{2}{*}{ Genetic region } & \multirow[b]{2}{*}{ Allele type } & \multirow[b]{2}{*}{ MF } & \multirow{2}{*}{$\frac{\mathrm{HW} \text { test }}{P \text { value }}$} & \multicolumn{3}{|c|}{ Narrow model } & \multicolumn{3}{|c|}{ Broad model } \\
\hline & & & & & & $N$ & $\chi^{2}$ test & $P$ value & $N$ & $\chi^{2}$ test & $P$ value \\
\hline rs1633445 & 18475150 & HTF9C (intron 10) & $\mathrm{T}=1 \mathrm{C}=2$ & 0.1667 & 0.4143 & 214 & 0.975 & 0.324 & 216 & 0.429 & 0.512 \\
\hline rs175174 & 18502108 & ZDHHC8 (intron 4) & $G=1 \quad A=2$ & 0.3328 & 0.2938 & 214 & 1.053 & 0.305 & 216 & 1.223 & 0.269 \\
\hline
\end{tabular}

Broad model, composed of Diagnostic and Statistical Manual IV (DSM-IV) schizophrenia, schizoaffective disorder and nonaffective psychotic disorders; HW, HardyWeinberg's test; MF, minor allele frequency; $N$, number of families; narrow model, only participants of schizophrenia fulfilling criteria of DSM-IV; SNP, single nucleotide polymorphisms. 
model) and 1.0161 (narrow model) for rs 1633445 , respectively, and 1.0138 (broad model) and 1.0107 (narrow model) for rs 175174 , respectively. Furthermore, no transmission distortions were discovered between gender and schizophrenia. The analogous rs175174 ratios for females were $1.0762(P=0.3172)$ and 1.0909 $(P=0.2175) ; \quad$ and $0.9714 \quad(P=0.5902)$ and 0.9667 $(P=0.5219)$ for males, using the narrow and broad models, respectively.

According to the subgrouping principle, there were 95 CPT deficit (undegraded or degraded CPT $\geq-2.5$ ) families for the undegraded group and 102 families for the degraded group. The CPT nondeficit (undegraded or degraded CPT $\gamma-2.5$ ) families were 75 for the undegraded group and 62 families for the degraded group. In total, we have 170 families with CPT data and 48 families without CPT data. A generalized estimating equation (GEE) model was performed by using SAS GENMOD procedure to compare the demographic data and clinical symptoms of the affected siblings of the missing 48 families with those of others. Most of the demographic data and clinical symptoms except the educational level were not significantly different between these two groups (data not shown). Though this sample with available CPT data was biased toward patients of higher educational level, it may have minimal influences on subsequent analysis because the indices of CPT were corrected for the educational level.

Significant results were shown when association analyses were performed between the SNP markers and the subgroup of schizophrenia with deficits in sustained attention (Table 3). The HTF9C SNP (rs1633445) showed significant association $(P<0.04)$ with the subgroup of schizophrenia having deficits in sustained attention assessed by undegraded CPT. The T allele is preferentially transmitted and is the risk allele type. No significant association was found for ZDHHC8 SNP (rs175174).

\section{Discussion}

Several schizophrenia candidate genes have been reported around the 22q11 region (Shifman et al., 2002;
Mukai et al., 2004; Chen et al., 2005). These candidate genes include catechol-O-methyltransferase (COMT) (chromosome position from 18303863 to 18331084) (Shifman et al., 2002), armadillo repeat gene deletes in velocardiofacial syndrome (ARVCF) (chromosome position from 18378863 to 18331973) (Chen et al., 2005), and ZDHHC8 (chromosome position from 18493918 to 18508528) (Mukai et al., 2004). The COMT was shown to have significant association with schizophrenia in Caucasians (Handoko et al., 2005), but we found no association in Taiwanese participants (Liou et al., 2001). As ZDHHC8 is only $163 \mathrm{~kb}$ from COMT, the negative association of ZDHHC8 with schizophrenia in this study is understandable. We conclude that these genes located at the 22q11 region may not be major susceptibility genes for schizophrenia in Taiwanese individuals.

The previous result supporting ZDHHC8 as a susceptibility gene for schizophrenia also showed the transmission distortions in women for the SNP marker of rs 175174 (Mukai et al., 2004). The transmitted-to-nontransmitted ratio was about $1.91(P=0.0005)$ in women, and about $0.98(P=0.88)$ in men in that study. Our result did not show transmission distortions for this SNP marker in either sex. This indicates the genetic structure of the Taiwanese family may be different from other ethnic groups (Race, 2005), and warrants further research.

Our results also indicate that the ZDHHC8 and its distal $5^{\prime}$-end of HTF9C genes may not form a good haplotype block. SNP rs1633445 of HTF9C and SNP rs175174 of ZDHHC8 have linkage disequilibrium $\left(\mathrm{D}^{\prime}\right)$ of 0.24 , suggesting that the chance for these two SNPs to compose a haplotype is low, possibly precluding the use of one SNP to represent the other.

Using 'deficit of sustained attention' as the endophenotype, defined as a degraded or undegraded CPT $\approx$ score of $\mathrm{D}^{\prime}<-2.5$, we found that the subgroup of schizophrenia with this endophenotype indicator of undegraded CPT had significant association with rs1633445 located at the intron 10 of HTF9C gene (Table 3). This was not found in the subgroup of schizophrenia families defined with the deficit in degraded CPT, possibly because degraded

Table 3 The association of the SNPs of ZDHHC8 and HTF9C genes and the subgroup of schizophrenia defined by endophenotype of deficit in sustained attention

\begin{tabular}{|c|c|c|c|c|c|c|c|c|c|c|c|c|c|}
\hline \multirow[b]{3}{*}{ СРT } & \multirow[b]{3}{*}{ SNP_ID } & \multicolumn{6}{|c|}{ Transmit } & \multicolumn{6}{|c|}{ FBAT } \\
\hline & & \multicolumn{3}{|c|}{ Narrow } & \multicolumn{3}{|c|}{ Broad } & \multicolumn{3}{|c|}{ Narrow } & \multicolumn{3}{|c|}{ Broad } \\
\hline & & $N$ & $\chi^{2}$ test & $P$ value & $N$ & $\chi^{2}$ test & $P$ value & $N$ & $Z$ & $P$ value & $N$ & $z$ & $P$ value \\
\hline Degraded & rs1633445 & 102 & 2.94 & 0.0864 & 104 & 1.6606 & 0.1975 & 24 & 1.620 & 0.1051 & 25 & 1.322 & 0.1861 \\
\hline$<-2.5$ & rs175174 & 102 & 0.0032 & 0.9546 & 104 & 0.1003 & 0.7515 & 37 & 0.423 & 0.672 & 39 & 0.676 & 0.4992 \\
\hline Undegra- & rs1633445 & 95 & 4.4802 & 0.0343 & 96 & 2.9305 & 0.0869 & 26 & 2.341 & 0.0192 & 27 & 2.057 & 0.0396 \\
\hline $\begin{array}{l}\text { ded } \\
<-2.5\end{array}$ & rs175174 & 95 & 0.513 & 0.4738 & 96 & 0.8825 & 0.3475 & 39 & 0.932 & 0.3514 & 41 & 1.069 & 0.2848 \\
\hline
\end{tabular}

$N$, number of families; SNP, single nucleotide polymorphisms. 
CPT demands mental activities other than pure sustained attention. This demonstrates the potential application of cognitive impairment to delineate subgroups of schizophrenia for genetic association studies. Owing to the small sample size and multiple-testing effect, our positive finding should be regarded as preliminary. This finding, however, does deserve replication by other study samples.

The HTF9C gene is for the first time demonstrating association with a subgroup of schizophrenia. The National Center for Biotechnology Information (NCBI) showed that the HTF9C may express in the brain tissue (http://www.ncbi.nlm.nih.gov/UniGene/ESTProfileViewer.cgi? $u g l i s t=H s .533019)$. The function role of HTF9C in the central nervous system is not clear. Using HTF9C antibody along with the antibodies of four other biomarkers can predict the outcome for breast cancer (Ring et al., 2006). Further studies on the function role of HTF9C gene in the brain may help in explaining its role in schizophrenia, especially in the subgroup of schizophrenia with deficit in sustained attention.

In conclusion, the regions of chromosome 22q11.21 containing the ZDHHC8 SNP (rs175174) and its $5^{\prime}$-distal SNP (rs1633445) of the HTF9C gene are not associated with a clinically defined and observable phenotype of schizophrenia. After subgrouping the phenotype of schizophrenia according to deficit and nondeficit of CPT, we found that the SNP representing HTF9C (rs1633445) was significantly associated in our study with the subgroup of schizophrenia defined by the endophenotype of deficit in undegraded CPT.

\section{Acknowledgements}

The authors acknowledge the help from the Department of Medical Research in National Taiwan University Hospital and the SNP genotyping work done by the National Genotyping Center (NGC). This study was supported by grants from the National Science Council, Taiwan (NSC-91-3112-B-002-011; NSC-92-3112-B-002019; NSC-93-3112-B-002-012; NSC-94-3112-B-002), the National Health Research Institute, Taiwan (NHRI90-8825PP; NHRI-EX91，92，93，94-9113PP; IRO1 MH59624-01), and the Department of Health, Taiwan (DOH94-TD-G-111-035).

\section{References}

American Psychiatric Association (1994). Diagnostic and statistical manual of mental disorders. 4th ed. Washington, DC: American Psychiatric Press.

Chen HY, Yeh Jl, Hong CJ, Chen CH (2005). Mutation analysis of ARVCF gene on chromosome $22 \mathrm{q} 11$ as a candidate for a schizophrenia gene. Schizophr Res 72:275-277.

Chen WJ (1999). Diagnostic interview for genetic studies (DIGS) Mandarin version 2.0. Taipei, Taiwan: Institute of Epidemiology, College of Public Health, National Taiwan University.

Chen WJ, Faraone SV (2000). Sustained attention deficits as markers of genetic susceptibility to schizophrenia. Am J Med Genet (Seminars in Medical Genetics) 97:52-57.
Chen WJ, Liu SK, Chang CJ, Lien YJ, Chang YH, Hwu HG (1998). Sustained attention deficit and schizotypal personality features in nonpsychotic relatives of schizophrenic patients. Am J Psychiatry 155: 1214-1220.

Chen WJ, Chang CH, Liu SK, Hwang TJ, Hwu HG (2004a). Sustained attention deficits in nonpsychotic relatives of schizophrenic patients: a recurrence risk ratio analysis. Biol Psychiatry 55:995-1000.

Chen WY, Shi YY, Zheng YL, Zhao XZ, Zhang GJ, Chen SQ, et al. (2004b). Case-control study and transmission disequilibrium test provide consistent evidence for association between schizophrenia and genetic variation in the 22q11 gene ZDHHC8. Hum Mol Genet 13:2991-2995.

Clayton D (1999). A generalization of the transmission/disequilibrium test for uncertain-haplotype transmission. Am J Hum Genet 65:1170-1177.

Cornblatt BA, Keilp JG (1994). Impaired attention, genetics, and the pathophysiology of schizophrenia. Schizophr Bull 20:31-46.

El-Husseini Ael D, Bredt DS (2002). Protein palmitoylation: a regulator of neuronal development and function. Nat Rev Neurosci 3:791-802.

Faul T, Gawlik M, Bauer M, Jung S, Pfuhlmann B, Jabs B, et al. (2005). ZDHHC8 as a candidate gene for schizophrenia: analysis of a putative functional intronic marker in case-control and family-based association studies. BMC Psychiatry 5:35.

Gabriel SB, Schaffner SF, Nguyen H, Moore JM, Roy J, Blumenstiel B, et al. (2002). The structure of haplotype blocks in the human genome. Science 296:2225-2229.

Glaser B, Schumacher J, Williams HJ, Jamra RA, lanakiev N, Milev R, et al. (2005). No association between the putative functional ZDHHC8 single nucleotide polymorphism rs175174 and schizophrenia in large European samples. Biol Psychiatry 58:78-80.

Handoko HY, Nyholt DR, Hayward NK, Nertney DA, Hannah DE, Windus LC, et al. (2005). Separate and interacting effects within the catechol-O-methyltransferase (COMT) are associated with schizophrenia. Mol Psychiatry 10: 589-597.

Horowitz A, Shifman S, Rivlin N, Pisante A, Darvasi A (2005). A survey of the $22 q 11$ microdeletion in a large cohort of schizophrenia patients. Schizophr Res 73:263-267.

Horvath S, Xu X, Lake SL, Silverman EK, Weiss ST, Laird NM (2004). Familybased tests for associating haplotypes with general phenotype data: application to asthma genetics. Genet Epidemiol 26:61-69.

Hwu HG (1999). Psychiatric diagnostic assessment. Manual of psychiatric diagnosis. 2nd ed. Taipei, Taiwan: Publication Committee, College of Medicine, National Taiwan University. pp 7-42.

Hwu HG, Chen CH, Hwang TJ, Liu CM, Cheng JJ, Lin SK, et al. (2002). Symptom patterns and subgrouping of schizophrenic patients: significance of negative symptoms assessed on admission. Schizophr Res 56: 105-119.

Hwu HG, Faraone SV, Liu CM, Chen WJ, Liu SK, Shieh MH, et al. (2005). Taiwan schizophrenia linkage study: the field study. Am J Med Genet $B$ Neuropsychiatr Genet 134:30-36.

Karayiorgou M, Gogos JA (2004). The molecular genetics of the 22q11associated schizophrenia. Brain Res Mol Brain Res 132:95-104.

Liou YJ, Tsai SJ, Hong CJ, Wang YC, Lai IC (2001). Association analysis of a functional catechol-O-methyltransferase gene polymorphism in schizophrenic patients in Taiwan. Neuropsychobiology 43:11-14.

Liu SK, Hsieh MH, Hwang TJ, Hwu HG, Liao SC, Lin SH, Chen WJ (2006). Re-examining sustained attention deficits as vulnerability indicators for schizophrenia: stability in the long term course. J Psychiatr Res 40: 613-621.

Lobo S, Greentree WK, Linder ME, Deschenes RJ (2002). Identification of a Ras palmitoyltransferase in Saccharomyces cerevisiae. J Biol Chem 277: 41268-41273.

Mukai J, Liu H, Burt RA, Swor DE, Lai WS, Karayiorgou M, Gogos JA (2004). Evidence that the gene encoding ZDHHC8 contributes to the risk of schizophrenia. Nat Genet 36:725-731.

Nuechterlein KH (1991). Vigilance in schizophrenia and related disorders. In: Steinhauer SR, Gruzelier JH, Zubin J, editors. Handbook of schizophrenia, Vol. 5: neuropsychology, psychophysiology and information processing. Amsterdam: Elsevier, pp. 397-433.

O'Connell JR, Weeks DE (1998). PedCheck: a program for identification of genotype incompatibilities in linkage analysis. Am J Hum Genet 63: 259-266.

Race E, and Genetics Working Group (2005). The use of racial, ethnic, and ancestral categories in human genetics research. Am J Hum Genet 77: 519-532.

Ring BZ, Seitz RS, Beck R, Shasteen WJ, Tarr SM, Cheang MC, et al. (2006). Novel prognostic immunohistochemical biomarker panel for estrogen receptor-positive breast cancer. J Clin Oncol 24:3039-3047. 
Rosvold HE, Mirsky AF, Sarason I, Bransome ED Jr, Beck LH (1956). A continuous performance test of brain damage. J Consul Psychol 20:343-350. Roth AF, Feng Y, Chen L, Davis NG (2002). The yeast DHHC cysteine-rich domain protein Akr1p is a palmitoyl transferase. J Cell Biol 159:23-28.

Saito S, Ikeda M, Iwata N, Suzuki T, Kitajima T, Yamanouchi Y, et al. (2005). No association was found between a functional SNP in ZDHHC8 and schizophrenia in a Japanese case-control population. Neurosci Lett 374: 21-24.

Shifman S, Bronstein M, Sternfeld M, Pisante-Shalom A, Lev-Lehman E, Weizman A, et al. (2002). A highly significant association between a COMT haplotype and schizophrenia. Am $J$ Hum Genet 71: 1296-1302. 